\title{
PARÂMETROS CINÉTICOS DE PRODUÇÃO DE GÁS IN VITRO DE DIFERENTES GENÓTIPOS DE CAPIM-ELEFANTE (Pennisetum purpureum SCHUM.)
}

\author{
Janderson Aguiar Rodrigues ${ }^{1}$ \\ Joadil Gonçalves de Abreu ${ }^{2}$ \\ Matheus Lima Correa Abreu ${ }^{3}$ \\ Lucas Matheus Barros Assis ${ }^{4}$ \\ Natalia Tousube da Rocha ${ }^{5}$ \\ Vicente Batista de Souza Júnior ${ }^{6}$ \\ Maxuel Fellipe Nunes Xavier ${ }^{7}$
}

\begin{abstract}
RESUMO
Objetivou-se neste trabalho avaliar modelos matemáticos comparando os parâmetros cinéticos na produção de gás in vitro em diferentes genótipos de capim-elefante. $\mathrm{O}$ delineamento foi inteiramente casualizado com três repetições com cinco tratamentos que foram os genótipos de capim-elefante (BRS Canará, Napier, Vruckwona, Cameroon e CNPGL 93-41-1) com idade de maturidade de rebrota aos 45 dias. $\mathrm{O}$ inóculo ruminal foi obtido de dois bois Nelores machos inteiros mantidos em piquetes formados por Urochloa brizantha cv. Marandu, no período seco do ano. As incubações foram consecutivas mensurando as leituras de pressão de produção de gás realizadas em $2 ; 4 ; 6 ; 8 ; 10 ; 12 ; 19 ; 24 ; 36 ; 48 ; 60 ; 72 ; 84$ e 96 horas. A determinação da cinética de produção de gases foi testada por dois modelos não lineares que são: Monomolecular e o Logístico. Foram estimados o volume final $\left(V_{f} / \mathrm{mL}\right)$, taxa média de produção de gás $\left(k / \mathrm{h}^{-1}\right)$, o tempo de latência de produção $(L / \mathrm{h})$ e o tempo médio de digestão $\left(\mathrm{TMD} / \mathrm{h}^{-1}\right)$. Os modelos testados, o Logístico com latência apresentou melhor ajuste para todos os perfis das cultivares. A cultivar BRS Canará e CNPGL 93-41-1 e Wrockwona, apresentam estimativas dos parâmetros cinéticos de produção de gás semelhantes e melhores aos comparados as cultivares Cameroon e Napier.
\end{abstract}

Palavras-chave: forragem, modelos matemáticos, produção de gás.

\section{KINETIC PARAMETERS OF IN VITRO GAS PRODUCTION OF DIFFERENT GENOTYPES OF ELEPHANT-GRASS (Pennisetum purpureum SCHUM.)}

\footnotetext{
ABSTRACT

The aim of this study was to evaluate mathematical models comparing the kinetic parameters in the production of in vitro gas in different genotypes of elephant grass. The design was entirely randomized with three repetitions with five treatments that were the elephant grass

${ }^{1}$ Doutorando no Programa de Pós Graduação em Ciência Animal pela Universidade Federal de Mato Grosso/Campus Cuiabá, Cuiabá-MT, Correspondência: janderson_zoo@hotmail.com

2 Professor, Doutor, Departamento de Zootecnia e Extensão Rural, Universidade Federal de Mato Grosso/Campus Cuiabá, Cuiabá-MT,joadil@terra.com.br

${ }^{3}$ Pós-doutorando, Universidade Estadual Norte Fluminense Darcy Ribeiro, Campos dos Goytacazes-RJ, matheus.zoot@yahoo.com.br

${ }^{4}$ Mestrando, Programa de Pós Graduação em Ciência Animal, Universidade Federal de Mato Grosso/Campus Cuiabá, Cuiabá-MT, zootecnialucas@gmail.com

${ }^{5}$ Graduanda em Zootecnia, Instituto Federal de Educação, Ciência e Tecnologia de Mato Grosso/Campus São Vicente, Campo Verde-MT, natalirocha2@hotmail.com

${ }^{6}$ Mestrando no Programa de Pós Graduação em Zootecnia pela Universidade Federal de Goiás/Campus Samambaia, Goiânia-GO. vicentebatista941@gmail.com

${ }^{7}$ Graduando em Agronomia no Instituto Federal de Educação, Ciência e Tecnologia de Mato Grosso/Campus São Vicente, Campo Verde-MT. maxuelfellipe90@gmail.com

Rodrigues JA, Abreu JG, Abreu MLC, Assis LMB, Rocha NT, Souza Jr, VB, Xavier MFN. Parâmetros cinéticos de produção de gás in vitro de diferentes genótipos de Capim-Elefante (Pennisetum purpureum Schum.). Vet. e Zootec. 2021; v28: 001-008.
} 
genotypes (BRS Canará, Napier, Vruckwona, Cameroon and CNPGL 93-41-1) with age of regrowth at 45 days. The ruminal inoculum was obtained from two whole male Nelores oxen kept in pickets formed by Urochloa brizantha cv. Marandu in the dry period of the year. The incubations were consecutive, measuring the gas production pressure readings in $2 ; 4 ; 6 ; 8 ; 10$; $12 ; 19 ; 24 ; 36 ; 48 ; 60 ; 72 ; 84$ and 96 hours. The determination of gas production kinetics was tested in two non-linear models: Monomolecular and Logistic. Where the final volume $(\mathrm{Vf} / \mathrm{mL})$, average gas production rate $\left(\mathrm{k} / \mathrm{h}^{-1}\right)$, production lage time $(\mathrm{Lag} / \mathrm{h})$ and average digestion time $(\mathrm{MDT} / \mathrm{h})$ were estimated. The models tested, the logistic with latency showed better fit for all crop profiles. The BRS Canará and CNPGL 93-41-1 and Wrockwona cultivars present estimates of kinetic parameters of gas production similar and better than those of Cameroon and Napier cultivars.

Keywords: fodder, gas production, mathematical models.

\section{PARÁMETROS CINÉTICOS DE LA PRODUCCIÓN DE GAS IN VITRO DE DIFERENTES GENOTIPOS DE PASTO ELEFANTE (Pennisetum purpureum SCHUM.)}

\section{RESUMEN}

El objetivo de este trabajo fue evaluar modelos matemáticos comparando los parámetros cinéticos en la producción de gas in vitro en diferentes genotipos de pasto elefante. El diseño fue completamente al azar con tres repeticiones con cinco tratamientos que fueron los genotipos de pasto elefante (BRS Canará, Napier, Vruckwona, Camerún y CNPGL 93-41-1) con una madurez de rebrote a los 45 días. El inóculo ruminal se obtuvo de dos novillos Nelores machos mantenidos en potreros formados por Urochloa brizantha cv. Marandu, en el período seco del año. Las incubaciones fueron consecutivas midiendo las lecturas de presión de producción de gas realizadas en $2 ; 4 ; 6 ; 8 ; 10 ; 12 ; 19 ; 24 ; 36 ; 48 ; 60 ; 72 ; 84$ y 96 horas. La determinación de la cinética de producción de gas se probó en dos modelos no lineales, que son: Monomolecular y Logístico. Donde se estimaron el volumen final $(\mathrm{Vf} / \mathrm{mL})$, la tasa de producción de gas promedio $\left(k / h^{-1}\right)$, el tiempo de latencia de producción $(L / h)$ y el tiempo de digestión promedio (TMD/ $\left.\mathrm{h}^{-1}\right)$. Los modelos probados, el Logístico con latencia, presentaron el mejor ajuste para todos los perfiles de cultivares. El cultivar BRS Canará y CNPGL 93-411 y Wrockwona, presentan estimaciones de los parámetros cinéticos de producción de gas similares y mejores a los de los cultivares Camerún y Napier.

Palabras clave: forraje, modelos matemáticos, producción de gas.

\section{INTRODUÇÃO}

A utilização de forrageiras para corte, as capineiras constituem uma das alternativas propostas para aliviar o problema de falta de forragem na época mais crítica do ano. Entre as espécies mais usadas para tal objetivo destaca-se o capim-elefante (Pennisetum purpureum Schum.) devido ao seu elevado potencial de produção de matéria seca e à sua boa aceitabilidade pelo gado. O capim-elefante é uma gramínea tropical de elevada capacidade de acumulação de matéria seca (MS), com o avanço da maturidade de tecidos vegetais da forrageira e a digestibilidade da MS estão intimamente associados, interferindo decisivamente na utilização como alimento para os ruminantes (1).

As cultivares de capim-elefante foram melhoradas para ser produtivas, grande quantidade de massa ( $\mathrm{MS} \mathrm{ha}^{-1}$ /ano), e por ser uma gramínea bastante utilizada na nutrição de 
ruminantes, principalmente gado de leite, que além da grande quantidade massa de forragem, mas também em qualidade de proteína bruta (CP) e de fibra em detergente neutros (NDF) e em carboidratos solúveis. Conforme o avanço da idade de rebrota do capim, afeta nos teores de fibra da planta. Aumento da maturidade da planta, folhas e hastes geralmente leva ao aumento do conteúdo da parede celular (e menor digestibilidade da matéria seca) devido ao aumento da proporção do caule, espessamento da parede celular ou menor conteúdo de proteínas e outros solúveis celulares (2). Segundo Van Soest (3), a fibra é um carboidrato estrutural usado como fonte de energia pelos micro-organismos do rúmen e tem sido usada para caracterizar alimentos e para estabelecer limites máximos de ingredientes nas rações.

A técnica acumulativa de gases tem sido muito usual nos laboratórios de nutrição de ruminantes, e permite avaliar os parâmetros cinéticos de produção de gás, oriundo da fração degradável, o volume de gás assintótico, taxas de digestão e latência $(4,5)$. Dessa forma, pode ser utilizado para determinar a importância de algumas dessas diferentes frações do alimento, em fornecer energia aos micro-organismos (6). Tilley e Terry (7) iniciaram os estudos baseados na simulação dos processos de digestão ruminal, e avaliaram a degradabilidade e a cinética de fermentação ruminal, pelo método de produção de gás in vitro, em 1963.

Consiste no desenvolvimento de uma dinâmica matemática ou modelo para avaliar a relação de taxa de digestão para utilização de forragem. Requer que o desaparecimento de alimentos do trato digestivo e ser separado em componentes e reações que podem ser conceituadas (8). A integração de parâmetros cinéticos para degradação e passagem permite a extensão da degradação dos diferentes nutrientes dos alimentos para estimado (9).

O modelo de crescimento de gás assume-se que primeiro a massa celular é produzida em proporção ao substrato digerido e segundo a produção de gás ocorre em proporção à quantidade de substrato digerido (4). Dessa forma, os modelos matemáticos devem ser empregados em estudos da natureza dos perfis de degradação ou de produção de gases, utilizado como ferramenta quantitativa importante para estimação do valor nutricional dos alimentares empregados na alimentação de ruminantes (10).

Portanto, objetivou-se neste trabalho avaliar modelos matemáticos comparando os parâmetros cinéticos na produção de gás in vitro em diferentes genótipos de capim-elefante.

\section{MATERIAL E MÉTODOS}

O trabalho foi conduzido no Laboratório de Nutrição Animal da Universidade de Mato Grosso - FAAZ Campus Cuiabá. As amostras de capim foram coletadas no campo experimental da Empresa Mato-grossense de Pesquisa, Assistência e Extensão Rural (EMPAER), localizada no município de Cáceres - MT, com coordenadas geográficas: 16 `43'42" S e 57 40'51" W, a 118 metros de altitude. Segundo Köppen e Geiger (11), a classificação climática é do tipo Aw, ou seja, clima tropical com estação chuvosa no verão, caracterizado por duas estações bem definidas (inverno seco e verão chuvoso), onde as precipitações de chuvas são superiores a $1800 \mathrm{~mm}$ ao ano. O delineamento experimental foi inteiramente casualizado com três repetições sendo que cada incubação foi uma repetição, com cincos tratamentos que foram os genótipos de capim-elefante (BRS Canará, Napier, Vruckwona, Cameroon e CNPGL 93-41-1) com idade de maturidade de rebrota aos 45 dias. As amostras de capim-elefante foram coletadas e secas à temperatura de $55^{\circ} \mathrm{C}$ sob circulação de ar por 72 horas. Após a secagem, as amostras foram moídas $(1 \mathrm{~mm})$ em moinho de faca tipo Wiley e armazenadas em potes impermeáveis de plástico.

O líquido ruminal foi coletado a partir das 6:00 horas da manhã na Fazenda Experimental FAAZ/UFMT de dois bois machos inteiros com aproximadamente $450 \pm 50 \mathrm{~kg}$ de peso corporal, canulados no rúmen, aprovado pelo Comitê de Ética no uso de animais (Processo $\mathrm{n}^{\mathrm{o}}$ 23108.207702017-76), suplementados em piquetes formados por Urochloa 
brizantha cv. Marandu, no período seco do ano. O inóculo foi filtrado e armazenados em garrafas térmicas pré-aquecidas a $39{ }^{\circ} \mathrm{C}$.

Foram pesados $0,5 \pm 0,05 \mathrm{~g}$ de amostras pré-secadas, e colocadas em frascos tipo "penicilina" (100 mL). Aos frascos com amostras foram adicionados $40 \mathrm{~mL}$ da solução tampão de McDougal (12), em seguida 10mL de líquido ruminal (relação 1:4 inóculo e solução tampão). Antes da vedação dos frascos, o espaço livre dos mesmos foi saturado com $\mathrm{CO}_{2}$. Os frascos foram fechados com tampa de borracha e lacrados com anilhas de alumínio. As leituras de pressão foram realizadas em $2 ; 4 ; 6 ; 8 ; 10 ; 12 ; 19 ; 24 ; 36 ; 48 ; 60 ; 72 ; 84$ e 96 horas de incubação, por meio de um transdutor (PressDATA 800) de pressão em psi. As leituras de pressão dos gases em psi, se converteu-se em volume de gás em $\mathrm{mL}$, onde os valores de volume total de produção de gás foram expressos em $\mathrm{mL} / 0,1 \mathrm{~g}$ de MS.

Para determinação da cinética de produção de gases foram testados dois modelos matemáticos não lineares que são: Modelo 1 - Monomolecular (Mod.1) e o Modelo 2 Logístico (Mod. 2) (13):

$$
\begin{aligned}
& \left(V_{t}=V_{f}[1 \exp (-k t)] M o d .1\right. \\
& \left(V_{t}=V_{f} \exp \{-\exp [1+k e(L-t)]\} M o d .2\right.
\end{aligned}
$$

Onde $V_{t}$ é a produção de gás $(\mathrm{mL})$ no tempo de incubação $\mathrm{t}(\mathrm{h}) ; V_{f}(\mathrm{~mL}) k$ é a taxa média de produção de gás $\left(\mathrm{h}^{-1}\right)$ e $L$ (Mod.2) é tempo de latência de produção (h) que está relacionada ao tempo de hidratação e colonização das amostras pelos micro-organismos. $\mathrm{O}$ tempo médio de digestão TMD $\left(\mathrm{h}^{-1}\right)$ para cada modelo foi estimado pela recíproca da taxa de produção de gás $(1 / k, \operatorname{Mod} .1)$, e no caso da Mod. 2 foi somado o tempo de latência $(1 / k+$ $L)$.

As análises foram realizadas pelo programa estatístico $\mathrm{R}$ utilizando-se o pacote nlme (14). A escolha do melhor modelo para cada cultivar de capim elefante foi conduzida pelo critério de informação de Akaike corrigido para pequenas amostras (AICc) (15), de maneira que o modelo que apresentou menor valor foi o escolhido como de melhor ajuste ao perfil de produção de gás. As análises dos perfis de produção de gás foram realizadas por meio do procedimento não linear misto (PROC NLMIXED) do SAS (University Edition). Os valores dos parâmetros foram estimados com intervalo de confiança de $95 \%$.

\section{RESULTADOS DISCUSSÃO}

Dentre os modelos testados, o modelo logístico com latência apresentou melhor ajuste para todos os perfis dos genótipos. As equações foram comparadas com base nos critérios de informação (AICc) utilizando o de menor valor conforme apresentado na Tabela 1. Dessa forma, o modelo permiti uma melhor interpretação quantitativa dos dados da produção acumulativa de gás. O modelo logístico deduz que a taxa de produção de gás é proporcional à atividade microbiana, representado pela quantidade de gás já produzida, e à concentração de substrato $(4,16)$.

O modelo que apresentou o melhor ajuste, originou estimativas paramétricas por ponto e entre valores observados que permite inferência confiáveis. Um modelo convergente descreve os dados da produção de gás ao longo do tempo, sendo essencial para avaliar os resultados dos estudos de cinética de produção de gases (16).

Na Tabela 2 são apresentados os valores estimados de cada parâmetro. A produção de gás assintótica, de acordo com intervalo de confiança, foi semelhante entre as cultivares BRS Canará e CNPGL 93-41-1, sendo os maiores valores encontrados para este parâmetro, por outro lado, a cultivar Cameroon apresentou o menor valor para $V f$. A produção acumulativa de gás é o somatório da digestão dos carboidratos não fibroso e fibroso. 
Tabela 1. Valores do critério de Akaike corrigidos dos modelos testados para os diferentes genótipos de capim-elefante.

\begin{tabular}{cccccc}
\hline & \multicolumn{5}{c}{ Genotypes } \\
\cline { 2 - 6 } Model & BRS Canará & Napier & Wruckwona & Cameroon & CNPGL 93-41-1 \\
\cline { 2 - 6 } & 113,5 & 105,8 & 105,4 & 109,4 & 114 \\
Monomolecular & 68,6 & 61,4 & 69,6 & 66,8 & 69,4 \\
\hline
\end{tabular}

AICc, critério de informação de Akaike corrigido do modelo; (Mod.1) Monomolecular; (Mod.2) Logística.

A taxa de produção de gás $(k)$ de todas as cultivares estudadas foram semelhantes BRS Canará, CNPGL 93-41-1, Wrockwona, Cameroon e Napier, respectivamente.

A latência ( $L$ ) foi menor para a cultivar Wruckwona, tendo as demais cultivares apresentado tempo de latência semelhante Cameroon, CNPGL 93-41-1, BRS Canará e Napier, respectivamente (Tabela 2). A latência demonstra o tempo gasto para a microbiota aumentar para níveis comparáveis com maior inoculo (6). O tempo necessário para a preparação da fixação microbiana entrar em contato com o substrato, dando início a fermentação (17). Essa diferença na latência está relacionada às características físico-químicas dos genótipos. $\mathrm{O}$ TMD observado para as cultivares foi maior para o Napier comparado as demais cultivares CNPGL 93-41-1, Wrockwona, Cameroon e BRS Canará.

Tabela 2. Valores médios com intervalos de confiança (IC 95\%) para o volume final de produção de gás $V f(\mathrm{~mL})$, taxa de digestão $k\left(\mathrm{~h}^{-1}\right)$, latência ou tempo de latência $L\left(\mathrm{~h}^{-1}\right)$ e tempo médio de digestão TMD (h) para os diferentes genótipos de capim-elefante.

\begin{tabular}{cccccc}
\hline \multirow{2}{*}{ Parâmetros } & \multicolumn{5}{c}{ Genótipos } \\
\cline { 2 - 6 } & BRS Canará & Napier & Wruckwona & Cameroon & CNPGL 93-41-1 \\
\cline { 2 - 5 } & \multicolumn{5}{c}{ Média \pm IC 95\% } \\
\hline$V$ & $116,0 \pm 2,6$ & $110,3 \pm 2,4$ & $110,4 \pm 2,8$ & $99,8 \pm 2,7$ & $111,2 \pm 2,8$ \\
$K$ & $0,022 \pm 0,002$ & $0,020 \pm 0,001$ & $0,021 \pm 0,002$ & $0,021 \pm 0,002$ & $0,022 \pm 0,002$ \\
$L$ & $9,5 \pm 1,0$ & $8,8 \pm 0,9$ & $7,0 \pm 1,1$ & $11,0 \pm 1,3$ & $10,9 \pm 1,2$ \\
TMD & 54,2 & 60,1 & 55,8 & 55,8 & 56,7 \\
\hline
\end{tabular}

Médias seguidas das mesmas letras na coluna não diferiram significativamente pelo teste de Tukey (p <0.05).

Isso pode ser um reflexo das características físico-químicas do substrato ou as condições desfavoráveis causadas ao inóculo ruminal durante a preparação para a incubação. Portanto, a aplicação de estimativas de retardamento in vitro em situações in vivo pode exigir algum cuidado.

Na Figura 1 são apresentados os perfis de produção acumulativa de gás, com um comparativo no volume assintótico $(\mathrm{mL})$ de gás produzido entre todos genótipos, tendo como destaque a cultivar BRS Canará com maior volume final. 

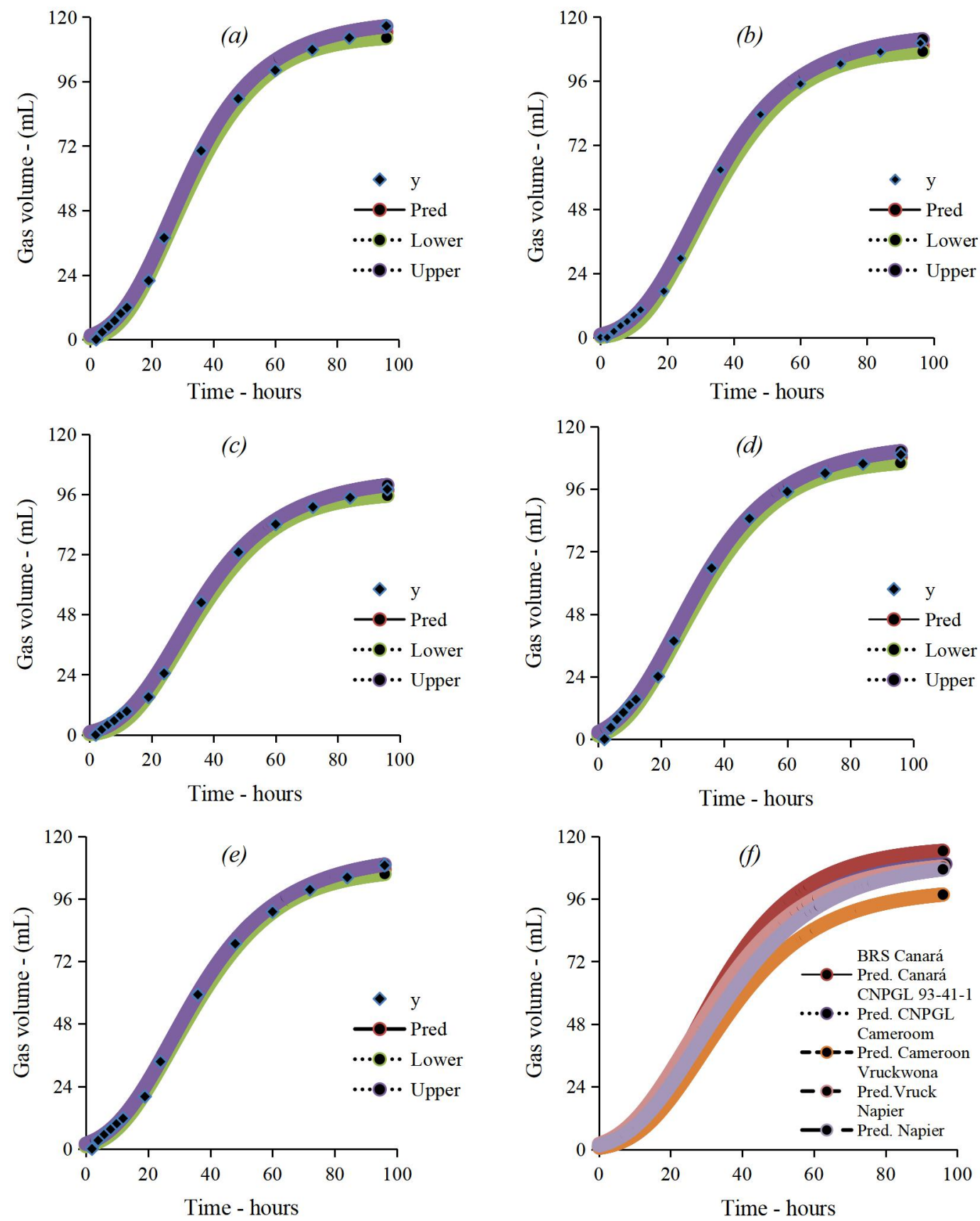

Figura 1. Perfis ajustado para cada genótipo de capim-elefante. Dados observados (y); valor predito (Pred) e intervalo de confiança (IC 95\%) entre o inferior e superior; (a) BRS Canará; (b) CNPGL 93-41-1; (c) Cameroom; (d) Vruckwona; (e) Napier; $(f)$ Comparação de produção de gás dos genótipos.

\section{CONCLUSÃO}

O modelo Logístico com Latência apresentou o melhor ajuste para todos os perfis de produção de gás in vitro, possibilitando as estimativas confiáveis dos parâmetros. Os genótipos BRS Canará e CNPGL 93-41-1 e Wrockwona, apresentam estimativas dos 
parâmetros cinéticos de produção de gás semelhantes e melhores aos comparados das cultivares Cameroon e Napier.

\section{AGRADECIMENTOS}

A Capes-Fapemat pela bolsa de estudos e a EMPAER Cáceres-MT pela parceria.

\section{REFERÊNCIAS}

1. Campos FP, Lanna DPD, Bose MLV, Boin C, Sarmento P. Degradabilidade do capimelefante em diferentes estágios de maturidade avaliada pelo método in vitro/gás. Sci Agric. 2002;59(2):217-8. doi: https://doi.org/10.1590/S0103-90162002000200003.

2. Wilson JR. Cell wall characteristics in relation to forage digestion by ruminants. J Agric Sci. 1994;122(2):173-9. doi: https://doi.org/10.1017/S0021859600087347.

3. Van soest PJ. Nutritional ecology of the ruminant [Internet]. Ithaca: Constock Publishing Associates; 1994 [citado 17 Nov 2020]. Disponível em: https://books.google.com.br/books?hl=pt-BR\&lr=\&id=mwUu6PL1UgC\&oi=fnd\&pg $=$ PP13\&dq $=$ Van + soest + PJ. + Nutritional + ecology + of + the + ru minant. + Ithaca: + Constock + Publishing + Associates,$+1994 . \&$ ots $=$ DQPAv6HfNC\&sig $=0$ VL duw-7Ke515Bxas4i1zsuEUsY

4. Schofield P, Pitt RE, Pell AN. Kinetics of fiber digestion from in vitro gas production. J Anim Sci. 1994;72(11):2980-91. doi: https://doi.org/10.2527/1994.72112980x.

5. Brutti DD, De Paula NF, Zervoudakis JT, Cabral LS, Fonseca MA, Macedo BG, et al. Effects of tannins and monensin on the modulation of in vitro ruminal fermentation and ammonia production of nitrogen-fertilized and non-fertilized Urochloa brizantha cv. Marandu. Grassland Sci. 2019;65(2):1-8. doi: https://doi.org/10.1111/grs.12221.

6. Pell AN, Schofield P. Computerized monitoring of gas production to measure forage digestion in vitro. J Dairy Sci. 1993;76(4):1063-73. doi: https://doi.org/10.3168/jds.S0022$\underline{0302(93) 77435-4 .}$

7. Tilley JMA, Terry RA. A two-stage technique for the in vitro digestion of forage crops. Grass Forage Sci. 1963(2);18:104-7. doi: https://doi.org/10.1111/j.13652494.1963.tb00335.x.

8. Mertens DR, Ely LO. Relationship of rate and extent of digestion to forage utilization - a dynamic model evaluation. J Anim Sci. 1982;54(4):895-905. doi: https://doi.org/10.2527/jas1982.544895x.

9. López S, France J, Gerrits WJ, Dhanoa MS, Humphries DJ, Dijkstra J. A generalized Michaelis-Menten equation for the analysis of growth. J Anim Sci. 2000;78(7):1816-28. doi: https://doi.org/10.2527/2000.7871816x.

10. Vieira RAM, Fernandes AM. A importância de estudos quantitativos associados à fibra para a nutrição e a alimentação de ruminantes. Rev Bras Zootec [Internet]. 2006 [citado 
11 Nov 2020];35:258-32. Disponível em: https://www.researchgate.net/profile/RicardoAugusto-

Vieira/publication/317082040 A Importancia de Estudos_Quantitativos_Associados a Fibra para a Nutricao e a Alimentacao de Ruminantes/links/59247897a6fdcc44430 bdbe0/A-Importancia-de-Estudos-Quantitativos-Associados-a-Fibra-para-a-Nutricao-e-aAlimentacao-de-Ruminantes.pdf

11. Köppen W, Geiger R. Klimate der Erde. Gotha: Verlag Justus Perthes; 1928.

12. Mcdougall EI. Studies on ruminant saliva. 1. The composition and output of sheep's saliva. Biochem J. 1948;43(1):99-109. doi: https://doi.org/10.1042/bj0430099.

13. López S, France J, Dhanoa MS, Mould F, Dijkstra J. Comparison of mathematical models to describe disappearance curves obtained using the polyester bag technique for incubating feeds in the rumen. J Anim Sci. 1999;77(7):1875-88. doi: https://doi.org/10.2527/1999.7771875x.

14. Pinheiro JC, Bates DM. Mixed-effects models in sand S-PLUS. New York: Springer; 2000 .

15. Akaike HA. New look at the statistical model identification. IEEE Trans Automat Contr. 1974;19(6):716-23. doi: https://doi.org/10.1109/TAC.1974.1100705.

16. Beuvink JMW, Kogut J. Modeling gas production kinetics of grass silages incubated with buffered ruminal fluid. J Anim Sci. 1993;71(4):1041-5. doi: https://doi.org/10.2527/1993.7141041x.

17. Krishnamoorty U, Soller H, Steingass H, Menke KH. A comparative study on rumen fermentation of energy supplements in vitro. J Anim Physiol Anim Nutr (Berl). 1991;65(1):28-35. doi: https://doi.org/10.1111/j.1439-0396.1991.tb00237.x.

Recebido em: 23/03/2021

Aceito em: 29/09/2021 